\title{
Performance Analysis of Uplink Packet Schedulers in Cellular Networks with Relaying
}

\author{
Desislava C. Dimitrova ${ }^{1}$, Hans van den Berg ${ }^{1,2}$, and Geert Heijenk ${ }^{1}$ \\ 1 University of Twente, Postbus 217, 7500 AE Enschede, The Netherlands \\ \{d.c.dimitrova, geert.heijenk\}@ewi.utwente.nl \\ 2 TNO ICT, The Netherlands \\ J.L.vandenBerg@ewi.utwente.nl
}

\begin{abstract}
Deployment of intermediate relay nodes in cellular networks, e.g. UMTS/ HSPA, has been proposed for service enhancement, which is of particular importance for uplink users at the cell edge suffering from low power capacity and relatively poor channel conditions. In this paper, we propose and investigate a number of uplink packet scheduling schemes deploying the relay functionality in different ways. Using a combined packet and flow level analysis capturing the specifics of the scheduling schemes and the random behavior of the users (initiation and completion of flow transfers), the performance of the various schemes is evaluated and compared to a reference scenario where relaying is not used. The main performance measures considered in our study are the instantaneous data rate, the energy consumption and the mean flow transfer time. Interestingly, considering flow transfer times, it is found that the use of relay nodes is not only particularly beneficial for users at the cell edge but also has a strongly positive effect on the performance of users at locations close to the base station.
\end{abstract}

\section{Introduction}

In the last decade UMTS (Universal Mobile Telecommunications System) evolved from an on-paper standard to a full scale technology adopted by many operators around the globe. The system continues to advance towards improved radio resource efficiency and higher data rates with the HSPA (High Speed Packet Access) technologies being the latest upgrade. For the downlink, from base station to mobile, the HSDPA (HighSpeed Downlink Packet Access) technology has been standardized by 3GPP in [1]. Alternatively, [2] introduces the EUL (Enhanced Uplink) for the uplink, from mobile to base station. In EUL, the channel resource is shared among all active users. Channel access is organized by the base station (BS) according to a particular scheduling scheme operating on a time scale of $2 \mathrm{~ms}$ (TTI - Transmission Time Interval).

The key resource in EUL is the total power budget at the base station which is shared among the mobile stations (MSs). A mobile station is an EUL compatible device which often operates on batteries and as such has limited transmit power. Depending on its distance to the BS a MS can or cannot use the total available resource on its own. If it can, system throughput is optimized by single transmission during a time slot (TTI) [8]. Alternatively, if a MS cannot fully use the total available resource, simultaneous transmissions by multiple MSs are a better choice [6]. 
In recent years many studies concentrated on EUL performance evaluation of various scheduling schemes for EUL, [7|4] to mention some. In principle, independently of the scheme, remote MSs suffer from high path loss and thus low data rates. Some authors, see [10], propose service in parallel due to users' limited power capacity. However, this only improves resource utilization but not the offered service. Placing a relay station (RS) can positively influence data rates by breaking a long communication path MS-BS into two shorter paths, MS-RS and RS-BS namely. The idea of relaying is rather attractive, however its implementation is trivial since it poses new implementation decisions such as where to set the relay or what scheduler to apply. As each decision has several possible outcomes the number of potential implementations rapidly grows. Selecting an optimal solution requires identifying main implementation choices as well as their advantages and disadvantages. Most importantly, before any research, we need to show that relaying can improve performance.

The application of relaying has been researched for a range of cellular and computer networks. [12] discusses a downlink scenario with relays placed at $2 / 3$ of the cell radius. In the presence of interference the author shows, for a single MS, that sending via the relay is beneficial in terms of packet errors and delays. The authors of [9] have chosen for a LTE (Long Term Evolution) network with changing number of MSs but evaluate only outage probability. Furthermore, the paper benefits from considering scheduling which adapts to channel conditions but lacks realism since unlimited flow sizes are assumed. Other studies which show the advantages of relaying for OFDMA networks are [11[14], while [13] and [3] concentrate on WiMAX networks.

In this paper we show the potential performance benefits of relaying for EUL, with focus on the resulting modifications on packet scheduling. In particular, we discuss four relay-enabled Round Robin schedulers and a reference scheme which does not use relaying. The schemes are compared with respect to a range of performance measures such as received powers, energy consumption, instantaneous rates and mean flow transfer times. Additionally, we show how RS characteristics such as transmit power and position influence MS performance. In summary, the most prominent contributions of our research are: incorporating the impact of flow level dynamics in terms of randomly changing number of active users; diversity of schedulers with relaying as opposed to the few options discussed by other authors; and combined assessment of both MS performance and RS specifics.

The paper continues as follows. First, in Section 2, we briefly discuss the relaying concept and describe the scheduling schemes considered in this paper. The model description and analysis appear in Section 3 and Secion 4 respectively. Section 5 presents our findings of the performance evaluation. Finally, Section 6 summarizes our work.

\section{Relay-Enabled Round Robin Scheduling Schemes}

We first briefly recall the idea of relaying in a cellular system and introduce the required notation. Next, the scheduling schemes considered in this paper are described.

Relaying allows us to reduce signal degradation due to path loss by breaking up a long path into two short ones. However, these gains are against the cost of an additional transmission of the data at the relay node. In a relay-enabled system a MS chooses 


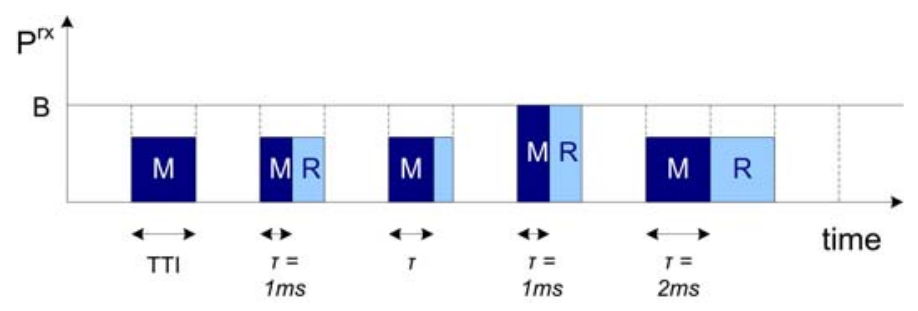

Fig. 1. Scheduling schemes: OBO, SOBO, SoptOBO, SidOBO and ExOBO

between direct communication to the BS (direct path MS-BS) and sending via the relay (relay path MS-RS-BS), depending on the data rates that can be realized on these paths. Note that the relay path consists of two sub-paths - MS-RS and RS-BS, and the data rate realized on the relay path depends on the achievable data rates on the two sub-paths.

Each (sub-)path is characterized by a set of transmission parameters: the distance between the communicating devices $d_{z z}$, the path loss $L_{z z}$, the transmit power $P_{z z}^{t x}$, the duration of a transmission opportunity $\tau_{z z}$ and the realized data rate $r_{z z}$ during a transmission opportunity. The index $z z$ refers to the specific (sub-)path, i.e. $m s$ for the direct path from MS to BS, $m r$ for the sub-path from MS to RS, and $r s$ for the sub-path from RS to BS. The transmission times $\tau_{m r}$ and $\tau_{r s}$ are scheduler specific; their sum is denoted by $\tau=\tau_{m r}+\tau_{r s}$. The relations between the transmission parameters are further discussed in Section 4 . We will now continue to introduce the specific scheduling schemes considered in our study.

\section{Scheduling Schemes}

Scheduling decisions are taken by the BS for which a relay behaves as a stationary MS that needs to be scheduled. Several approaches towards incorporating relay transmissions into scheduling are possible. We consider only Round Robin (RR) type of schedulers where mobile users are served one-by-one (OBO), independently of their channel conditions. Studies among which [10]8] show that OBO is inefficient in resource utilization when remote users with limited power capacity are served. However, a relay is closer to the MSs and it is more likely that a single MS is capable to fully use the available resources. In our study we consider four variants of relay-aware OBO

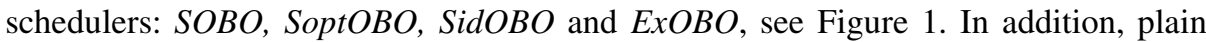
$O B O$ is considered as a reference scheme in which a MS always transmits directly to the base station independently of its location in the cell (and the duration of a single transmission opportunity equals one TTI, i.e. $\tau_{m s}=2 m s$. In the other four schemes a MS can select the direct or the relay path, depending on its location relative to the BS and the RS.

Extended $O B O(\mathrm{ExOBO})$ assigns (in the case an indirect path is chosen) a single TTI to the transmissions on both sub-paths, i.e. $\tau_{m r}=\tau_{r s}=2 m s$. Therefore, for the service of a particular mobile station in a single round of the RR scheme the base station sets aside 2 TTIs meaning $\tau=4 m s$. Transmissions on a direct path are assigned one TTI.

In the shared $O B O(\mathrm{SOBO})$ scheme transmissions on direct and indirect paths are assigned one TTI, i.e. $\tau=2 \mathrm{~ms}$. In the case of an indirect path, the single TTI is divided 


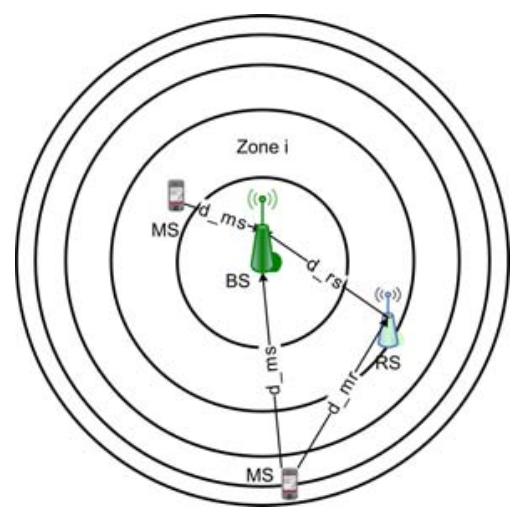

Fig. 2. Single cell model with cell division in zones. The distance between MS-BS, MS-RS and RS-BS are denoted as $d_{m s}, d_{m r}$ and $d_{r s}$, respectively.

into two equal intervals of $1 \mathrm{~ms}$ and MS and RS both receive one interval to transmit, i.e. $\tau_{m r}=\tau_{r s}=1 \mathrm{~ms}$. Assigning fixed-length transmission times of one TTI for direct and indirect paths eases implementation, but the static subdivision for indirect paths is inefficient when the achievable rates on the sub-paths differ.

Optimized SOBO (SoptOBO) also uses one TTI to serve a single MS, both for direct and indirect paths (i.e. $\tau=2 m s$ ). However, for indirect paths, it selects the transmission times on the sub-paths MS-RS and RS-BS such that the same amount of data is transferred, i.e. $\tau_{m r} r_{m r}=\tau_{r s} r_{r s}$. Although SoptOBO maximizes the resource utilization it is rather challenging for implementation. The selection of the specific transmission times for the sub-paths requires complex functionality in the base station.

Ideal SOBO (SidOBO) is the last relay-aware scheduling scheme we consider in our study. It is like SOBO but assumes ideal forwarding conditions, namely unlimited RS transmit capacity and interference budget. Under these assumptions, the relay is eliminated as a potential bottleneck. The scheme is more of a theoretical design rather than a practical solution, and provides us some notion of best-case performance.

\section{Model}

Our model consists of a single cell with EUL users (MSs) generating data traffic. In order to differentiate in the location of MSs we divide the cell in $K$ zones with equal area, see Figure 2. Each zone is characterized by its distance to the base station $d_{i}$, measured from the outer edge of the zone, and a corresponding path loss $L_{i}, i=1 \cdots K$. All MSs within the same zone $i$ are assumed to have the same distance $d_{i}, i=1 \cdots K$ to the base station. The distribution of (active) MSs over the zones is represented by the system state vector $\underline{n} \equiv\left(n_{1}, n_{2}, \cdots n_{i}, \cdots n_{K}\right)$, where $n_{i}$ is the number of currently active users (MSs) located in zone $i$.

Mobile stations become active, at uniformly distributed positions in the cell, according to a Poisson process with rate $\lambda$, and have to transfer a file with mean size $F$ (the file 
size has an exponential distribution). Hence, due to the equal area zones, all zones have the same file transfer initiation rate (or: arrival rate) $\lambda_{i}=\lambda / K$. Active MSs keep their position during the file transfer. A MS chooses between direct transmission or via the relay, depending on its location. All mobile stations have the same maximum transmit power capacity $P_{\max }^{t x}$. Depending on their location either the maximum transmit power or a lower power is used, i.e. $P^{t x} \leq P_{m a x}^{t x}$, such that a MS maximizes its utilization of the available budget $B$.

In our study a relay is a device with one basic functionality, namely resending data from MS to BS. Such simple design keeps the cost down and still provide improved performance to the MSs.

In this paper we assume multiple relay stations on a concentric circle around the base station, such that BS, RS and MS are on a straight line 1 . A fixed path selection policy based on distance is adopted according to which all MSs located beyond the relay, i.e. $d_{m s}>d_{r s}$, transmit via the indirect path and in all other cases, i.e. $d_{m s} \leq d_{r s}$, direct transmission is chosen. An ideal switching mechanism between transmitting and receiving at the RS is assumed as the same frequency band is used.

At both BS and RS limited channel resource, budget $B$, is assumed. Given noise rise $\eta$ and constant thermal noise $N$, the shared budget $B$ at the BS is derived: $B=\eta \times N$. The same budget $B$ is set also for the RS, with the exception of SidOBO for which the RS budget is unlimited. The other interference component we account for is the self-interference, i.e. the own signal parts which travel via secondary (reflected) paths. Intentionally disregarding important factors such as inter- and intra-cell interference allows us to identify the effect relaying has on performance. However, we realize that such factors may have negative effects on the performance gains.

\section{Analysis}

Relaying has two opposite effects on performance (excluding effects outside the scope of this paper): on the one hand it enables a higher received power level (increasing the data rate), but on the other hand forwarding at the RS requires an additional transmission of the data (decreasing effective data rates). Apart from that performance is influenced by the varying number of active users in the cell. In order to account for these effects we apply a combined packet and flow level analysis. For each scheduler we start with calculating received powers from which subsequently instantaneous data rates can be derived. Next we consider the flow level dynamics - flow initiation and flow completion - and model the system at flow level by a continuous time Markov chains (CTMC) with transmission rates derived from the instantaneous data rates and the system state $\underline{n}$. From the steady state distribution of the Markov chain the mean flow transfer time of a MS in a particular zone can be obtained. Our combined approach exhibits several advantages above other existing approaches. Starting at the packet level allows us to catch

\footnotetext{
${ }^{1}$ In fact, this assumption requires unlimited number of relays on the concentric circle but it can be shown that this ideal situation can be very well approximated by 8 or $10 \mathrm{RS}$. In principle, the analysis in this paper can easily be extended to any other assumption concerning the number and position of the RSs. However, for the purpose of the present paper, this specific choice suffices.
} 
specifics of scheduling scheme and environment. Applying Markov models supports the modeling of a real network where the number of active users constantly changes. The approach is also rather scalable since changing the scheduler or the environment only ask for recalculation of the instantaneous rate at the packet level.

\subsection{Received Powers}

According to the propagation law, the received power $P^{r x}$ is a direct result of applied transmit power $P^{t x} \leq P_{\max }^{t x}$ and path loss $L(d)$. Furthermore, the maximum possible received power of any MS is limited by the available budget $B$ resulting in: $P^{r x}=\min \left(P^{t x} / L(d) ; B\right)$. The assumed path loss model, taken from the Okumura-Hata model, is given by $L(d)=123.2+35.2 \log 10(d)$ (in $\mathrm{dB}$ ) with $d$ the distance in kilometer.

In Section 2 we explained how each scheduling scheme organizes its transmissions. Furthermore, when relay is used, we should differentiate between the received powers $P^{r x}(B S)$ and $P^{r x}(R S)$ at the base station and the relay station, respectively.

When a relay is used in SOBO, SidOBO and ExOBO the slower sub-path limits the transmission. Therefore, the transmit powers at MS and RS are chosen such that the received powers at RS and $\mathrm{BS}$ are equal $\left(P^{r x}(R S)=P^{r x}(B S)\right)$ and determined by the minimum of the achievable received powers on both sub-paths. In the case of SoptOBO $P^{r x}(R S)$ is in general not equal to $P^{r x}(B S)$ since the scheme deploys different data rates on the two relay sub-paths. Note that SidOBO differs from the rest in the higher maximum transmission powers of RS it uses. The scheme-specific received powers at the BS can be given as:

$$
P_{i}^{r x}(B S)= \begin{cases}P_{i, m s}^{r x} & \text { for OBO and direct path in other schedulers } \\ \min \left(P_{i, m r}^{r x}, P_{r s}^{r x}\right) & \text { for relay path in SOBO, SidOBO and ExOBO } \\ P_{i, r s}^{r x} & \text { for relay path in SoptOBO }\end{cases}
$$

\subsection{Instantaneous Rate}

The instantaneous rate $r$ of a particular MS represents its realized data rate when the MS is scheduled. Hence, $r$ can be defined as the amount of unique data transferred during the period $\tau$ when the BS serves that MS. Note that how much unique data is transferred is determined by how long the MS sends, i.e. $\tau_{m r}$. The dependency of the instantaneous rate on the other transmission parameters is thus given by:

$$
r_{i}=\frac{r_{c h i p}}{E_{b} / N_{0}} * \frac{P_{i}^{r x}}{N+\omega P_{i}^{r x}} * \frac{\tau_{i, m r}}{\tau},
$$

In Equation $2 i=1 \cdots K$ indicates zone number, $r_{c h i p}$ is the system chip rate and $E_{b} / N_{0}$ is the energy-per-bit to noise ratio. The parameter $\omega$ is used to account for reflected signals.

Each scheduler uses different policy for the selection of the total transmission time $\tau$ and the MS transmission times $\tau_{i, m r}$, see Section 2 After substitution the expressions of the scheduler specific instantaneous rates become: 


$$
r_{i}= \begin{cases}r_{i, m s} & \text { for OBO and direct path in others } \\ \min \left(r_{i, m r}, r_{i, r s}\right) * \frac{1}{2} & \text { for relay path in SOBO, SidOBO and ExOBO } \\ \frac{r_{i, m r} * \tau_{i, m r}}{\tau} & \text { for relay path in SoptOBO }\end{cases}
$$

In OBO and for all MSs selecting direct path $\tau_{m r}=\tau$ holds. SOBO, SidOBO and ExOBO assign to the mobile station only half of the total time $\tau$, since the factor $1 / 2$ in Equation (3). Finally, the more generic expression for SoptOBO follows from the strategy to adapt the transmission opportunities $\tau_{i, m r}$ according to the MS's zone.

\subsection{Flow Dynamics}

The actual rate a MS receives is lower or equal to the instantaneous rate $r_{i}$ and depends on the state $\underline{n}$ of the system and on the number of users $n$ in particular. This new rate we term state-dependent throughput $R_{i}(\underline{n})$ and it differs per scheduler. In all schemes but ExOBO the state-dependent throughput can be derived from $R_{i}(\underline{n})=r_{i} / n$. The difference for ExOBO originates in the different number of TTIs assigned to MSs using the direct and the relay path - one and two TTIs respectively. Hence, for $R_{i}(\underline{n})$ we can write:

$$
R(\underline{n})= \begin{cases}\frac{r_{i}}{n+n_{\text {relay }}} & \text { for MSs using direct path } \\ \frac{2 r_{i}}{n+n_{\text {relay }}} & \text { for MSs using relay path }\end{cases}
$$

where $n_{\text {relay }}$ is the number of MSs which make use of the relay station. Note that $r_{i}$ is difference for each path according to Equation (3).

The changes in the system state $\underline{n}$ (due to flow initiations and completions) are described by CTMC of $K$ dimensions with transition rates $\lambda_{i}$ and $R(\underline{n})$. The particular form of the Markov chain is scheduler specific via $R(\underline{n})$. A more detailed description of a related CTMC can be found in [4]. All schemes but ExOBO are a multi-class M/G/1 processor sharing (PS) model whose steady state distribution is readily available, e.g. [5]. For the ExOBO model we implemented a Markov chain simulator which based on large number of state transitions, i.e. 1 million, provides us with the steady state distribution. Subsequently, we can derive performance measures such as mean flow transfer times $T_{i}$ :

$$
T_{i}=\frac{1}{r_{i} F} * \frac{1}{1-\rho}
$$

where $i, i=1 \cdots K$ is zone number, $\rho$ the system load and $F$ the mean file size.

\subsection{Energy Consumption}

The retransmission of data on the relay path suggests higher energy consumption $E$ compared to direct transmission, given both have the same total duration $\tau$. However, $E$ is an absolute measure and does not account for the fact that different schemes might transfer different amount of data. To compensate we use the energy-per-bit $E_{b i t}$ measure which incorporates the details on data traffic and is given by:

$$
E_{b i t, i}=\frac{E_{i}}{r_{i, m r} \tau_{m r}}=\frac{E_{i}}{r_{r s} \tau_{r s}}
$$


where $E_{i}, i=[1 \cdots K]$, is the energy consumption of a MS in zone $i$. The product $\tau_{m r} *$ $r_{i, m r}$ gives the unique amount of data transferred on the relay path, which is a scheduler specific value.

\section{Numerical Results on Relaying}

In this section we present a quantitative evaluation of performance at both packet and flow level for the discussed scheduling schemes. We are particularly interested in comparing performance based on measures such as received powers, energy consumption, instantaneous rates and mean flow transfer times. Special attention is given to the impact of flow level dynamics. Most results are generated from mathematical analysis with small part coming from simulations, i.e. flow level analysis of ExOBO. Simulations are carried out with a generic simulator developed in Matlab for deriving the steady-state distribution of multi-dimensional Markov chains.

\subsection{Parameter Settings}

In the numerical experiments we apply system chip rate $r_{\text {chip }}$ of $3840 \mathrm{kchips} / \mathrm{s}$, thermal noise level $N$ of $-105.66 \mathrm{dBm}$ and noise rise target $\eta$ of $6 \mathrm{~dB}$, see [6]. For SidOBO the available budget $B$ at the RS is unlimited. Self-interference of $10 \%$ is considered, i.e. $\omega=0.1$.

The cell is divided in $K=10$ zones with equal area2, see Figure 2, Given a cell radius of $2 \mathrm{~km}$ we applied straightforward link budget calculations to determine the zone radii and the path losses. By default relay stations are located in zone 3 with some experiments explicitly saying when this changes. Both MS and RS has maximum transmit power $P_{\max }^{t x}=0.125$ Watt. EUL flows are taken with an $E_{b} / N_{0}$ target of $5 \mathrm{~dB}$ [6] and mean file size is $F=1000 \mathrm{kbit}$. The call arrival rate is set to $\lambda=0.4 \mathrm{calls} / \mathrm{sec}$.

\subsection{Received Power Levels}

In order to build up good understanding of the impact relaying has on schedulers, we confine the discussion of received powers to OBO, SOBO and ExOBO. The received powers at the BS as a function of the distance are presented in Figure 3(a) for a range of positions of the RSs. As expected, relaying increases received powers but the position of the RS has strong impact on the gain. Moving the RS towards the edge of the cell lowers the gain - effect caused by increased distance $d_{r s}$ between RS and BS and fewer MSs using the relay.

Recall that transmission on the relay path can be limited by both relay and mobile station, which is illustrated by the graphs for RSs in zone 1 and 2 in Figure 3(a). Typically signal degrades with distance as we can see from the graph of direct transmission, i.e. OBO. However, when the RS forms the bottleneck on the relay path, all MSs served by it are characterized by the same received powers at the BS which explains the unchanging graphs. Note that with RS in zone 3 and further this is always the case. If the MSs are the bottleneck and the received powers decrease in distance and differ per zone.

\footnotetext{
${ }^{2}$ Extensive numerical experiments showed that this granularity is sufficient for our purposes.
} 


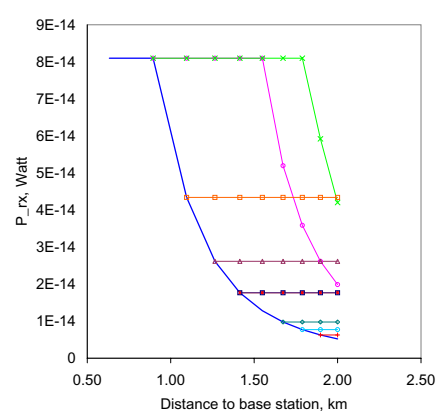

(a) Received powers

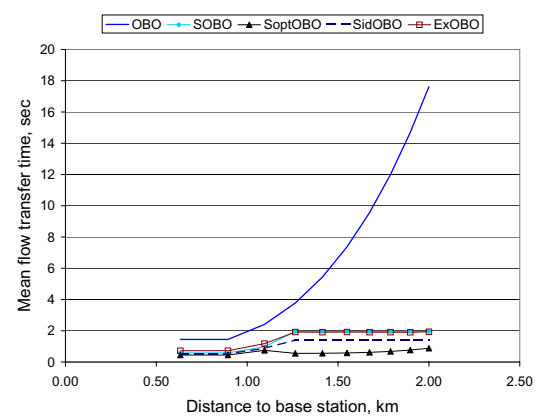

(c) Mean flow transfer times

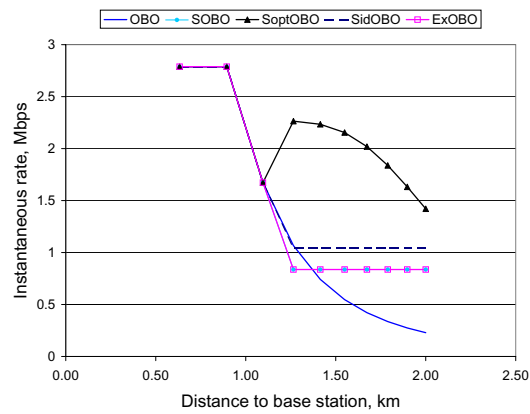

(b) Instantaneous data rates

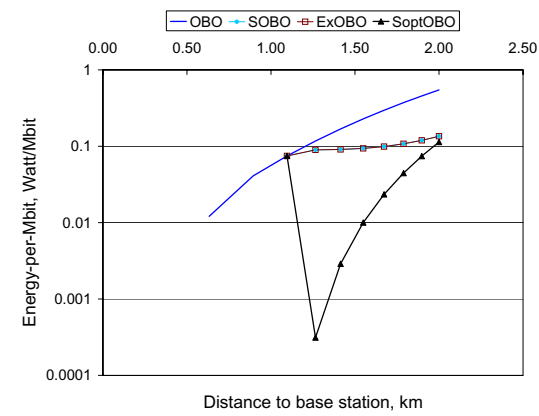

(d) Energy consumption

Fig. 3. Performance evaluation based on (a) received powers; (d) energy-per-bit consumption; (b) instantaneous rates; and (c) mean flow transfer times (flow level)

\subsection{Instantaneous Data Rates}

The instantaneous data rates generated by each of the five scheduling schemes are presented in Figure 3(b) Fixed path selection policy is applied. In all cases when direct transmission is used all schemes show the same performance. However, beyond zone 3 (after relay is used) the relay-enabled schemes outperform OBO meeting our expectations. The graphs of SOBO and ExOBO coincide, since both schemes have the same received powers at $\mathrm{BS}$ and use only half the transmission time $\tau$ to transfer unique data, see Equation (3). Contrary to our expectations not SidOBO is the best performing scheme but SoptOBO. Even if the RS does not limit transmissions, a MS can use only the available budget $B$ at the BS for the fixed time $\tau_{m r}$. SoptOBO circumvents the limitation by using adaptive transmission times.

The disadvantage of relay due to forwarding is well illustrated by the graphs of SOBO and ExOBO. Given our fixed path selection policy, MSs close behind the relay would be better if they could select the direct path. Furthermore, in OBO and SoptOBO rate degrades in distance since both schemes differentiate in users from different zones. Alternatively, given a relay in zone 3, all transmissions on the relay path in SOBO, $\mathrm{SidOBO}$ and ExOBO are limited by the relay which explains the flat graphs. 


\subsection{Mean Flow Transfer Times}

The dependency of mean flow transfer times of the distance to base station, for each of the schedulers, is presented in Figure 3(c). Some of the observations made for the instantaneous rates in Section 5.3 are confirmed but also new ones exhibit. In particular, Figure 3(c) shows that the gain achieved by using RSs is even more emphasized when we consider the mean flow transfer times as a performance measure. An interesting new observation is that even the performance of close-by MSs which use direct transmissions is improved as result of relaying. Higher data rates for remote MSs translate to lower system load (number of active MSs $n$ ), which is beneficial for all active MSs, see Equation (4), independently whether they use relay or not.

\subsection{Energy Consumption}

Figure 3(d) shows the energy-per-bit consumption as a function of distance. As so far, the differences in performance between the schedulers exhibit when a relay station is used. All relay-enabled schemes record lower $E_{b i t}$ than OBO due to their lower applied powers and higher achievable data rates. SoptOBO has the best performance since its optimal TTI division maximizes data rates. Energy consumption increases with distance as result of increasing transmit powers of the MSs. The effect of distance is largest for SoptOBO due to the flexible transmission times which imply that stations with high transmit powers send for a long period, see Section 2 . On the contrary, in SOBO and ExOBO the transmission times are fixed and the increase in energy consumption smoother.

\section{Conclusion}

We have compared the performance of four Round Robin packet schedulers for uplink transmission in a cellular network with relaying. As performance measures we used received powers at the base station, energy consumption, instantaneous data rates and mean flow transfer times. Analysis of the flow transfer times (taking into account that the number of active users varies over time) is enabled by a combined packet- and flow level analysis. Our study reveals the pros and cons of the four relay-aware scheduling schemes, and makes a comparison to the performance achieved in the case without relaying. As expected, relaying significantly improves the service offered to remote users with poor channel conditions. Interestingly, it is also beneficial for users which do not use the relay - an effect that can be seen only at the flow level. The latter observation supports our claim that the flow level dynamics have crucial influence on scheduling performance, and needs to be taken into account in performance comparisons.

As topics for further study we suggest investigating alternative relay-aware schedulers (e.g. with parallel transmissions), and examining the mutual relay influence in a more complex scenario with multiple cells.

\section{Acknowledgments}

The authors would like to thank Remco Litjens for the prolific discussions and for providing us with challenging new ideas. 


\section{References}

1. 3GPP TS 25.308. High Speed Downlink Packet Access (HSDPA); Overall Description

2. 3GPP TS 25.309. FDD Enhanced Uplink; Overall Description

3. Bel, A., Seco-Granados, G., Vicario, J.L.: The benefits of relay selection in wimax networks. In: ICT-MobileSummit 2008 (2008)

4. Dimitrova, D.C., van den Berg, H., Heijenk, G., Litjens, R.: Flow-level performance comparison of packet scheduling schemes for UMTS EUL. In: Harju, J., Heijenk, G., Langendörfer, P., Siris, V.A. (eds.) WWIC 2008. LNCS, vol. 5031, pp. 27-40. Springer, Heidelberg (2008)

5. Haverkort, B.: Performance of computer communication systems. John Wiley \& Sons Ltd., Chichester (1999)

6. Holma, H., Toskala, A.: HSDPA/HSUPA for UMTS. John Wiley \& Sons Ltd., Chichester (2006)

7. Liu, T., Mäder, A., Staehle, D., Everitt, D.: Analytic modeling of the UMTS enhanced uplink in multi-cell environments with volume-based best-effort traffic. In: IEEE ISCIT 2007, Sydney, Australia (October 2007)

8. Ramakrishna, S., Holtzman, J.M.: A scheme for throughput maximization in a dual-class CDMA system. In: ICUPC 1997, San Diego, USA (1997)

9. Reetz, E., Hockmann, R., Tonjes, R.: Performance study on cooperative relaying topologies in beyond 3g systems. In: ICT-MobileSummit 2008 (2008)

10. Rosa, C., Outes, J., Sorensen, T.B., Wigard, J., Mogensen, P.E.: Combined time and code division scheduling for enhanced uplink packet access in WCDMA. In: IEEE VTC 2004, Los Angeles, USA (Fall 2004)

11. Schoenen, R., Halfmann, R., Walke, B.H.: An fdd multihop cellular network for 3gpp-lte, May 2008, pp. 1990-1994 (2008)

12. Umlauft, M.: Relay devices in umts networks - effects on. In: Proceedings of the Fifth Annual Mediterranean Ad Hoc Networking Workshop, Med-Hoc-Net 2006 (2006)

13. Vidal, J., Munoz, O., Agustin, A., Calvo, E., Alcon, A.: Enhancing 802.16 networks through infrastructure-based relays. In: ICT-MobileSummit 2008 (2008)

14. Wei, H.Y., Ganguly, S., Izmailov, R.: Ad hoc relay network planning for improving cellular data coverage. In: Personal, Indoor and Mobile Radio Communications, 2004. PIMRC 2004, September 2004, vol. 2, pp. 769-773 (2004) 